\title{
COVID-19: una revisión de la evidencia en el ámbito pediátrico
}

\author{
COVID-19: A Review of the Available Evidence Regarding Pediatric \\ Population
}

Gina Nieto-Duarte ${ }^{1}$, Patricia Pichilingue ${ }^{2}$, Iván Aivasovsky-Trotta ${ }^{3}$, Marianna Castellanos-Fernandez ${ }^{4}$, Luis Gustavo-Celis ${ }^{5}$

\section{Resumen}

En el contexto de la pandemia por COVID-19, es importante la selección y el uso adecuado de los conocimientos nuevos y de aquellos adquiridos en situaciones históricas similares, para garantizar una correcta toma de decisiones en cuanto a la prevención, manejo y tratamiento de esta enfermedad en la población pediátrica. Desde inicios del 2020, la atención se ha focalizado en el control de la pandemia y en el manejo de los pacientes con esta enfermedad, cuya mayoría se encuentra en la población adulta. Sin embargo, recientemente se han observado cursos más severos de la enfermedad en pacientes pediátricos y lo que inicialmente se consideraba como una patología inofensiva ha generado mayores alertas en esta población por la presencia de complicaciones severas. Por lo anterior, la presente revisión busca determinar las últimas estrategias de prevención, diagnóstico y tratamiento avaladas y soportadas por la evidencia científica, de manera que se aporten las herramientas necesaria para garantizar un manejo adecuado y disminuir, en la medida de lo posible, los desenlaces fatales en la población pediátrica.

\footnotetext{
1. Médica general, Universidad de La Sabana. Pediatra, New York Medical College / St. Joseph Children's Hospital, NJ. Pediatra Nefróloga, Baylor College of Medicine/TCH Houston, Texas. Correo electrónico: ginanieto4@hotmail.com

ORCID: https://orcid.org/0000-0003-0190-2586

2. Médica General, Universidad Peruana Cayetano Heredia. Pediatra, New York Medical College / St. Joseph Children's Hospital, NJ. Infectóloga pediátrica, University of Texas Southwestern Medical Center. HIV y SIDA en Pediatría, St. Jude Children's Research Hospital. Correo electrónico: patriciapichilingue@yahoo.com ORCID: https://orcid.org/0000-0002-9142-4292

3. Facultad de Medicina, Universidad de La Sabana, Chía, Cundinamarca Correo electrónico: ivac94@gmail.com

ORCID: https://orcid.org/0000-0003-0320-2735

4. Facultad de Medicina, Universidad de La Sabana, Chía, Cundinamarca. Correo electrónico: mariannacastellanosf@gmail.com ORCID: https://orcid.org/0000-0001-5477-7200

5. Facultad de Medicina, Universidad de La Sabana, Chía, Cundinamarca Correo electrónico: luis.celis@unisabana.edu.co ORCID: https://orcid.org/0000-0002-0338-6258
} 
Palabras claves: COVID-19, síndrome respiratorio agudo severo 2, pediatría, manejo de enfermedades, prevención secundaria.

\section{Abstract}

In the context of the COVID-19 pandemic, the adequate selection and appropriate use of previously acquired knowledge from similar historical situations and the one acquired lately amid the pandemic is vital to guarantee correct decision-making regarding the prevention, management and treatment of this disease in the pediatric population. Since the beginning of 2020, attention has shifted to controlling the pandemic and managing patients with this disease, the majority being those in the adult population. However, more severe courses of the disease have recently been observed in pediatric patients, and what was initially considered to be a harmless pathology in this population has generated greater alerts due to the presence of severe complications. Therefore, this review seeks to determine the latest prevention, diagnosis and treatment strategies endorsed and supported by scientific evidence, in order to provide the necessary tools to guarantee proper management and to reduce, as far as possible, the fatal outcomes in the pediatric population.

Keywords: COVID-19, Severe acute respiratory syndrome coronavirus 2, Pediatrics, Disease Management, Secondary Prevention.

\section{Introducción}

La enfermedad originada en la provincia de Wuhan, China por el nuevo virus SARS$\mathrm{CoV}-2$ ha tenido una rápida progresión hasta derivar en una pandemia a mediados de Marzo (1). Esta ha sido responsable de más de 10 millones de casos y 470000 muertes asociadas (2). En muchos estudios a nivel mundial, se ha reportado que el COVID-19 afecta desproporcionalmente a adultos en comparación con nińos, ya que menos del 5 $\%$ de los casos son en población pediátrica (3-6).
En menores, se ha establecido una mayor tasa de casos asintomáticos y casos leves en comparación con adultos $(7,8)$. Se ha visto que un IMC para la edad mayor a 85 (sobrepeso) o 95 (obesidad), al igual que estados de inmunosupresión y preexistentes condiciones médicas complejas, son factores de riesgo para enfermedad severa $(7,9)$.

\section{Prevención}

Se debe hacer énfasis en el bienestar físico, la ausencia de comorbilidades, adecuado 
estado nutricional, salubridad y medidas de higiene como agentes mitigadores del contagio y esparcimiento de la enfermedad $(10,11)$. Asimismo, la higiene de manos es una medida efectiva que se parece disminuir la transmisión de las partículas y gotas que contienen el virus (12).

Los estados de malnutrición y las carencias de micro- y macronutrientes pueden tener un efecto negativo en el desenlace de los pacientes con COVID-19 ya que promueven estados proinflamatorios y comprometen el sistema inmunológico (13-20). No obstante, son necesarios más estudios para determinar la eficacia de la suplementación como estrategia terapéutica.

La vacunación como estrategia preventiva viene en desarrollo por la comunidad científica desde el inicio de la pandemia. La OMS registró más de 100 entidades que buscan desarrollar una vacuna, de las cuales 10 se encuentran en fase clínica. Se espera que esta sea la principal herramienta preventiva; sin embargo, a pesar de los esfuerzos, esta sigue siendo una posibilidad remota $(21,22)$.

\section{Presentaciones clínicas}

Casi la mitad de los casos cursan con cuadros leves y aproximadamente un cuarto de los casos son asintomáticos (8). Se ha visto que los pacientes pediátricos cursan con cuadros más leves que los adultos $(3,4)$.Por su parte, los pacientes que requieren ingreso a unidad de cuidados intensivos tienen alguna comorbilidad (obesidad, inmunosupresión, etc.) $(9,23)$.

Los síntomas más comunes son fiebre y tos seca $(3,4,7,8,24)$. Y en menor proporción otro espectro de síntomas (tabla 1) $(3,8,23)$.

Tabla 1. Espectro clínico de síntomas observados relacionados con la frecuencia de aparición en pacientes pediátricos con COVID-19.

\begin{tabular}{|c|c|}
\hline Síntoma & Frecuencia \\
\hline Fiebre & $36-41,5 \%$ \\
\hline Tos seca & $19-48 \%$ \\
\hline Odinofagia & $6 \%$ \\
\hline Congestión faríngea & $3-46,2 \%$ \\
\hline Disnea y taquipnea & $3-28 \%$ \\
\hline Síntomas gastrointestinales (vómito y diarrea) & $6-8 \%$ \\
\hline Desaturación oxígeno & $2 \%$ \\
\hline
\end{tabular}

Fuente: Adaptada de Ludvigsson JF y cols; Qiu H, Wu J y cos; Lu X, Zhang y cols (3, 8, 23). 
Los hallazgos radiológicos son las opacidades múltiples y en parche. En la tomografía lo más común es vidrio esmerilado, engrosamiento bronquial o lesiones inflamatorias pulmonares $(8,23)$.

\section{Diagnóstico y estratificación}

El diagnóstico de COVID-19 se realiza a través de hisopado nasofaríngeo o pruebas moleculares sanguíneas de reacción de cadena polimerasa (PCR); al igual que en muestras de esputo y lavado bronquio-alveolar $(3,24)$. También se utilizan métodos de secuenciación genética de SARS-CoV 2 en muestras del tracto respiratorio y sanguíneo (3), o PCR en heces o sangre y serología , pero no son de utilidad en la fase aguda de la infección (4).

Ahora bien, se realizan pruebas diagnósticas en aquellos que cumplan algún criterio epidemiológico y al menos dos clínicos (tabla 2) (25).

Tabla 2. Criterios epidemiológicos y clínicos.

\begin{tabular}{|l|l|}
\hline \multicolumn{1}{|c|}{ Epidemiológico } & \multicolumn{1}{|c|}{ Clínico } \\
\hline $\begin{array}{l}\text { Nińo que haya viajado a alguna región con epidemia en los últimos } \\
14 \text { días previos al inicio de los síntomas }\end{array}$ & Fiebre, fatiga, tos seca y síntomas respiratorios \\
\hline $\begin{array}{l}\text { Niños que hayan tenido contacto con pacientes con infección } \\
\text { confirmada en los últimos 14 días previos al inicio de los síntomas }\end{array}$ & Hallazgos radiológicos \\
\hline $\begin{array}{l}\text { Nińos que hayan tenido contacto con pacientes con fiebre o } \\
\text { síntomas respiratorios de comunidad con casos reportados en los } \\
\text { últimos 14 días previos al inicio de los síntomas }\end{array}$ & $\begin{array}{l}\text { En la fase temprana de la enfermedad, glóbulos } \\
\text { blancos normales o disminuidos } \\
\text { (linfopenia) }\end{array}$ \\
\hline $\begin{array}{l}\text { Dos o más casos fiebre o síntomas respiratorios en los últimos 14 } \\
\text { días en grupos pequeńos (salón de clase, familia, etc.) }\end{array}$ & No identificación de otro agente causal \\
\hline Recién nacido de madres con infección confirmada & \\
\hline
\end{tabular}

Fuente: Tomada de China National Clinical Research Center for Respiratory Diseases (25).

Se considera un caso confirmado aquellos sospechosos que cumplan los siguientes criterios (25):

\section{Test positivo para SARS-CoV 2 por} RCP en tiempo real

2. Secuenciación genética de SARS-CoV 2 del tracto respiratorio o muestra sanguínea

3. $\operatorname{IgM}$ e $\operatorname{Ig} G$ específico positiva
4. IgG sanguíneo específico que cambia de negativa a positiva, o que aumenta 4 veces el valor previo

5. Anticuerpos totales contra SARSCOV-2

Posterior al diagnóstico, se debe clasificar al paciente según la severidad de la enfermedad (tabla 3) (25). 
Tabla 3. Clasificación del paciente pediátrico con COVID-19.

\begin{tabular}{|c|c|}
\hline Clasificación & Características \\
\hline Infección asintomática & Test positivo para SARS-CoV 2 pero sin síntomas clínicos ni hallazgos radiológico \\
\hline $\begin{array}{l}\text { Infección respiratoria aguda } \\
\text { superior }\end{array}$ & $\begin{array}{l}\text { Solo presentan fiebre, tos, odinofagia, congestión nasal, fatiga, cefalea, mialgias, disconfort, sin } \\
\text { signos de neumonía en radiología de tórax ni de sepsis }\end{array}$ \\
\hline Neumonía leve & $\begin{array}{l}\text { Con o sin fiebre, con síntomas del tracto respiratorio superior (tos) con radiografía de tórax } \\
\text { sugestiva de neumonía viral, sin criterios de neumonía severa }\end{array}$ \\
\hline Neumonía severa & $\begin{array}{l}\text { Polipnea (> } 60 \text { respiraciones por minutos en menores de dos meses, }>50 \text { respiraciones por } \\
\text { minuto de } 2 \text { a } 12 \text { meses, }>50 \text { respiraciones por minutos en } 1 \text { a } 5 \text { años, o }>30 \text { respiraciones por } \\
\text { minuto en mayores } 5 \text { años). } \\
\text { Saturación de oxígeno }<92 \% \text {, disnea, cianosis o apnea intermitente. } \\
\text { Alteración estado de conciencia. } \\
\text { Rechazo en la alimentación o dificultad en la alimentación con signos de deshidratación. } \\
\text { Tomografía de tórax de alta resolución que muestra infiltrados bilaterales o multilobares, } \\
\text { progresión rápida de la enfermedad o efusión pleural. }\end{array}$ \\
\hline Críticos & $\begin{array}{l}\text { Aquellos que cumplen cualquiera de los siguientes criterios requieren cuidados intensivos: } \\
\text { Falla respiratoria que requiere ventilación mecánica. } \\
\text { Shock. } \\
\text { Falla multiorgánica. }\end{array}$ \\
\hline
\end{tabular}

Fuente: Tomada de China National Clinical Research Center for Respiratory Diseases (25).

\section{Tratamiento}

El tratamiento en la población pediátrica va dirigida al manejo de soporte, control de sintomatología y monitorización de posibles complicaciones (4). Para la fiebre, se recomienda el uso de medidas no farmacológicas, como bańos con agua tibia, y también medidas farmacológicas con antipiréticos incluyendo acetaminofén o medicamentos antiinflamatorios no esteroideos AINE (25).

Como parte del tratamiento sintomático, se puede usar expectorantes para evitar obstrucción del tracto respiratorio por secre- ciones, con acetil cisteína o solución salina normal (25).

Se debe garantizar un adecuado soporte respiratorio, con el uso de oxígeno suplementario a requerimiento de cada paciente, desde cánula nasal hasta ventilación mecánica invasiva cuando sea necesario. En la población pediátrica, el requerimiento de suplencia de oxígeno es menor a comparación con los adultos. Es necesario el uso oportuno de resucitación hídrica según los requerimientos y edad de cada paciente, e iniciar agentes vasoactivos cada vez que sea necesario (4). 
El uso de antivirales es controversial, dado que la evidencia es muy limitada; no hay estudios que demuestren seguridad y eficiencia en ninguno de los antivirales disponibles, y en los estudios actuales no suelen incluir menores de edad. Los más usado son el lopinavir/ritonavir, ribavirina, hidroxicloroquina e interferón alfa (9). El uso de esto está reservado para aquellos con casos severos o críticos (26).

El remdesivir, demostró mejorar las complicaciones respiratorias en los adultos. Ha demostrado disminuir la duración de síntomas y la estancia hospitalaria (27). La hidroxicloroquina, en periodos cortos de cinco días, ha sido empleado como inmunomodulador, en pacientes que no son candidatos a remdesivir o cuando este no está disponible. Ha demostrado disminución en la replicación viral ha reducido las exacerbaciones por neumonía y podría mejorar hallazgos radiológicos. La combinación con azitromicina no se recomienda por la prolongación del QT y el riesgo a muerte súbita en pacientes con arritmia subyacente $(7,9,26)$. El interferón alfa nebulizado, ha demostrado disminuir la carga viral y los síntomas (25).

El uso de glucocorticoides, en forma de pulsos de metilprednisolona, por 4 a 5 días, está indicado en casos severos, sin embargo, su uso está limitado a pacientes con soporte ventilatorio y hemodinámico, dadas las posibles reacciones adversas como necrosis avascular, osteoporosis y diabetes de novo $(4,25)$. Del mismo modo, el uso de inmu- noglobulina está indicado en casos severos según cada paciente y no hay estudios suficientes (25).

El ensayo clínico Recovery ha demostrado que la dexametasona en dosis de $6 \mathrm{mg}$ al día, por hasta 10 días, disminuiría la mortalidad a los 28 días en pacientes con ventilación mecánica invasiva y terapia de oxígeno sin soporte ventilatorio. El estudio incluyó también pacientes menores de 18 años y mujeres embarazadas (28).

\section{Referencias}

1. Millán-Oñate J, Rodriguez-Morales AJ, Camacho-Moreno G, Mendoza-Ramírez H, Rodríguez-Sabogal IA, Álvarez-Moreno C. A new emerging zoonotic virus of concern: the 2019 novel Coronavirus (COVID-19). Infectio. 15 de abril de 2020;24(3):187.

2. COVID-19 Map FAQ [Internet]. Johns Hopkins Coronavirus Resource Center. [citado 28 de junio de 2020]. Disponible en: https://coronavirus.jhu. edu/map-faq

3. Ludvigsson JF. Systematic review of COVID-19 in children shows milder cases and a better prognosis than adults. Acta Paediatr. junio de 2020;109(6):1088-95.

4. Zimmermann P, Curtis N. Coronavirus Infections in Children Including COVID-19: An Overview of the Epidemiology, Clinical Features, Diagnosis, Treatment and Prevention Options in Children. Pediatr Infect Dis J. mayo de 2020;39(5):355-68.

5. Bi Q, Wu Y, Mei S, Ye C, Zou X, Zhang Z, et al. Epidemiology and transmission of COVID-19 in 391 cases and 1286 of their close contacts in Shenzhen, China: a retrospective cohort study. Lancet Infect Dis. abril de 2020;S1473309920302875. 
6. Wu Z, McGoogan JM. Characteristics of and Important Lessons From the Coronavirus Disease 2019 (COVID-19) Outbreak in China: Summary of a Report of 72314 Cases From the Chinese Center for Disease Control and Prevention. JAMA. 7 de abril de 2020;323(13):1239.

7. Zachariah P, Johnson CL, Halabi KC, Ahn D, Sen AI, Fischer A, et al. Epidemiology, Clinical Features, and Disease Severity in Patients With Coronavirus Disease 2019 (COVID-19) in a Children's Hospital in New York City, New York. JAMA Pediatr. 3 de junio de 2020;e202430.

8. Qiu H, Wu J, Hong L, Luo Y, Song Q, Chen D. Clinical and epidemiological features of 36 children with coronavirus disease 2019 (COVID-19) in Zhejiang, China: an observational cohort study. Lancet Infect Dis. junio de 2020;20(6):689-96.

9. Shekerdemian LS, Mahmood NR, Wolfe KK, Riggs BJ, Ross CE, McKiernan CA, et al. Characteristics and Outcomes of Children With Coronavirus Disease 2019 (COVID-19) Infection Admitted to US and Canadian Pediatric Intensive Care Units. JAMA Pediatr [Internet]. 11 de mayo de 2020 [citado 28 de junio de 2020]; Disponible en: https:// jamanetwork.com/journals/jamapediatrics/fullarticle/2766037

10. Dechartres A, Boutron I, Trinquart L, Charles P, Ravaud P. Single-center trials show larger treatment effects than multicenter trials: evidence from a meta-epidemiologic study. Ann Intern Med. 2011;155(1):39-51.

11. McCormick M. Rats, Communications, and Plague: Toward an Ecological History. J Interdiscip Hist. julio de 2003;34(1):1-25.

12. Wolfe MK, Gallandat K, Daniels K, Desmarais AM, Scheinman P, Lantagne D. Handwashing and Ebola virus disease outbreaks: A randomized comparison of soap, hand sanitizer, and $0.05 \%$ chlorine solutions on the inactivation and removal of model organisms Phi6 and E. coli from hands and persistence in rinse water. Cameron DW, editor. PLOS ONE.
23 de febrero de 2017;12(2):e0172734.

13. Malavazos AE, Corsi Romanelli MM, Bandera F, Iacobellis G. Targeting the Adipose Tissue in COVID-19. Obesity. julio de 2020;28(7):1178-9.

14. Butler MJ, Barrientos RM. The impact of nutrition on COVID-19 susceptibility and long-term consequences. Brain Behav Immun. julio de 2020;87:534.

15. Elmadfa I, Meyer AL. The Role of the Status of Selected Micronutrients in Shaping the Immune Function. Endocr Metab Immune Disord - Drug Targets. 11 de noviembre de 2019;19(8):1100-15.

16. Zhang L, Liu Y. Potential interventions for novel coronavirus in China: A systematic review. J Med Virol. mayo de 2020;92(5):479-90.

17. Gasmi A, Noor S, Tippairote T, Dadar M, Menzel A, Bjørklund G. Individual risk management strategy and potential therapeutic options for the COVID-19 pandemic. Clin Immunol. junio de 2020;215:108409.

18. Semba RD, Tang AM. Micronutrients and the pathogenesis of human immunodeficiency virus infection. Br J Nutr. marzo de 1999;81(3):181-9.

19. Barazzoni R, Bischoff SC, Breda J, Wickramasinghe K, Krznaric Z, Nitzan D, et al. ESPEN expert statements and practical guidance for nutritional management of individuals with SARS-CoV-2 infection. Clin Nutr. junio de 2020;39(6):1631-8.

20. Naja F, Hamadeh R. Nutrition amid the COVID-19 pandemic: a multi-level framework for action. Eur J Clin Nutr [Internet]. 20 de abril de 2020 [citado 28 de junio de 2020]; Disponible en: http://www. nature.com/articles/s41430-020-0634-3

21. Mullard A. COVID-19 vaccine development pipeline gears up. The Lancet. junio de 2020;395(10239):1751-2.

22. Sun J, Zhuang Z, Zheng J, Li K, Wong RL-Y, Liu D, et al. Generation of a Broadly Useful Model for COVID-19 Pathogenesis, Vaccination, and Treatment. Cell. junio de 2020;S0092867420307418. 
23. Lu X, Zhang L, Du H, Zhang J, Li YY, Qu J, et al. SARS-CoV-2 Infection in Children. N Engl J Med. 23 de abril de 2020;382(17):1663-5.

24. Castagnoli R, Votto M, Licari A, Brambilla I, Bruno R, Perlini S, et al. Severe Acute Respiratory Syndrome Coronavirus 2 (SARS-CoV-2) Infection in Children and Adolescents: A Systematic Review. JAMA Pediatr [Internet]. 22 de abril de 2020 [citado 28 de junio de 2020]; Disponible en: https:// jamanetwork.com/journals/jamapediatrics/fullarticle/2765169

25. China National Clinical Research Center for Respiratory Diseases, National Center for Children's Health, Beijing, China, Group of Respirology, Chinese Pediatric Society, Chinese Medical Association, Chinese Medical Doctor Association Committee on Respirology Pediatrics, China Medicine Education Association Committee on Pediatrics, Chinese Research Hospital Association Committee on Pediatrics, et al. Updated diagnosis, treatment and prevention of COVID-19 in children: experts' consensus statement (condensed version of the second edition). World J Pediatr. junio de 2020;16(3):2329.

26. Chiotos K, Bassiri H, Behrens EM, Blatz AM, Chang J, Diorio C, et al. Multisystem Inflammatory Syndrome in Children During the Coronavirus 2019 Pandemic: A Case Series. J Pediatr Infect Dis Soc. 28 de mayo de 2020;piaa069.

27. Pharmacologic Treatments for Coronavirus Disease 2019 (COVID-19): A Review | Clinical Pharmacy and Pharmacology | JAMA | JAMA Network [Internet]. [citado 29 de junio de 2020]. Disponible en: https://jamanetwork.com/journals/jama/fullarticle/2764727

28. Effect of Dexamethasone in Hospitalized $\mathrm{Pa}$ tients with COVID-19: Preliminary Report | medRxiv [Internet]. [citado 29 de junio de 2020]. Disponible en: https://www.medrxiv.org/content/10.1101/2020.06.22.20137273v1 